\title{
Semi-automatic Face Image Finding Method, Which Uses the 3D Model of the Head for Recognising an Unknown Face
}

\author{
Olga Krutikova ${ }^{1}$, Aleksandrs Glazs ${ }^{2}$ \\ ${ }^{1,2}$ Riga Technical University
}

\begin{abstract}
In this paper, a semi-automatic facial recognition algorithm is proposed in case of an insufficient training set (profile, front, half-turn). The recognition algorithm uses a polygonal 3D model that is created from the base images. The control points, in the proposed method, are transferred from the base images onto the 3D model, and they are also placed on the new image from the examination set. Then, the 3D model is used to determine the rotation angle of the head on the image, and the distances between the control points are calculated on both the new image and the model images to determine which class the new image belongs to.
\end{abstract}

Keywords - 3D model, face recognition, insufficient training set, polygonal model.

\section{INTRODUCTION}

Facial recognition is one of the actual directions in the theory of pattern recognition. It has many tasks in a wide variety of fields, for example, in forensics, it is often necessary to identify a person, based on several a priori images (full face, profile etc.). A major problem that arises in pattern recognition is an insufficient volume of the training sample. This may be related to a lack of images in the training sample that correspond to various rotation angles of the head.

To solve the problem of insufficient information, it was proposed to use a transformation to the canonical position of the face [1], [2]. However, the effectiveness of such an approach depends on the position of the anthropometric points on the face and it is often associated with errors that occur if part of the face is darkened. In addition, problems arise with the perspective projections in case of two-dimensional images. That is why the information is needed about individual images at different angles of rotation of the head [3] or further expanding the training sample. Another approach is based on creating the three-dimensional model of the head, which could be used to acquire the information about the shape of the face and using this information in facial recognition [4], [5], [6]. In this case, the model can be rotated, thus expanding the insufficient training sample. This allows getting additional information about the different rotations of the head, since 3D models which contain shape [7] as well as texture are inferred from 2D images [8], [9]. However, it should be noted that it can only operate on specialised computing clusters since the algorithm is very computationally expensive [10], [11], [12].

In our previous research, we proposed an approach [13] for creating a polygonal 3D model of the head, constructed by using three base images (profile, front, half-turn), since this training set was insufficient, there was a method proposed for expanding it. Another method that was proposed to expand the training set [14], [15] included 4 new images of faces for each class. This approach also had its disadvantages: it could not precisely recognise the face at angles that were greater than 45 degrees. Therefore, in this paper, we propose a method for using the mentioned polygonal 3D model in the face recognition algorithm.

\section{THE PROPOSED METHOD}

A block diagram of the face recognition algorithm is shown in Fig. 1.

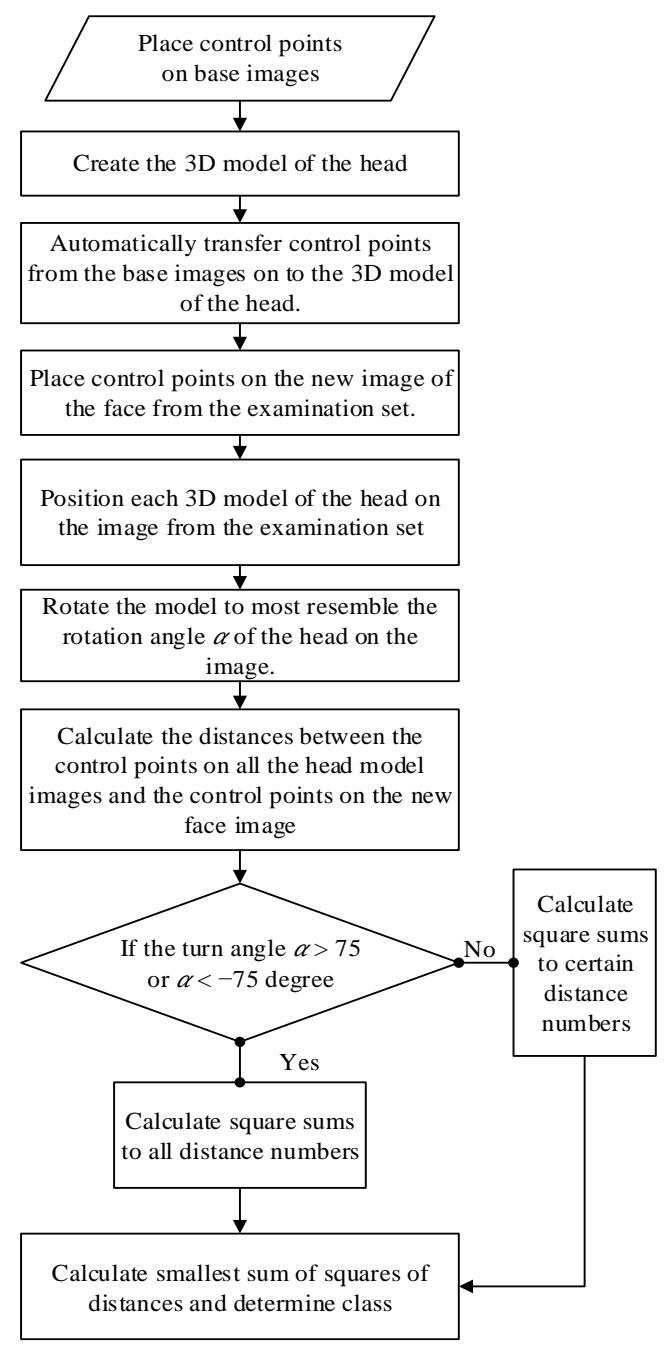

Fig. 1. Block diagram of the proposed algorithm. 
$2015 / 16$

The proposed method combines several image-processing algorithms. The method includes one existing 3D model creation algorithm [13], [15] and the proposed algorithms that are based on distance calculation between control points, and searching for minimum square sums in order to recognise new face images.

\section{A. Creation Algorithm of 3D Model of a Head}

The method includes one existing algorithm for creating a 3D model of a head, which is described in [13], and is modified in [15]. This algorithm is used to create different face models from a base training set (profile, half turn and full face images). Compared to previous methods, the proposed method uses only wireframe models without face texture.

\section{B. Automatic Transfer of Control Points from Base Images onto the 3D Model}

During the next step some of the control points (see Fig. 3. (a), (b)) were automatically transferred from base images (profile, full face) onto the 3D model of the head as different coloured points for each distance number (see Fig. 3, (c), (d)).

Searching for different colours is used to automatically detect point coordinates. If discovered colour is equal to the searched colour, then the pixel is marked and its coordinates $(x, y)$ are saved.

\section{Placing the Control Points on the New Image of the Face from the Examination Set}

In the next step, control points are placed on the new image from the examination set, in a similar way as with the points on the 3D model (See Fig. 2).

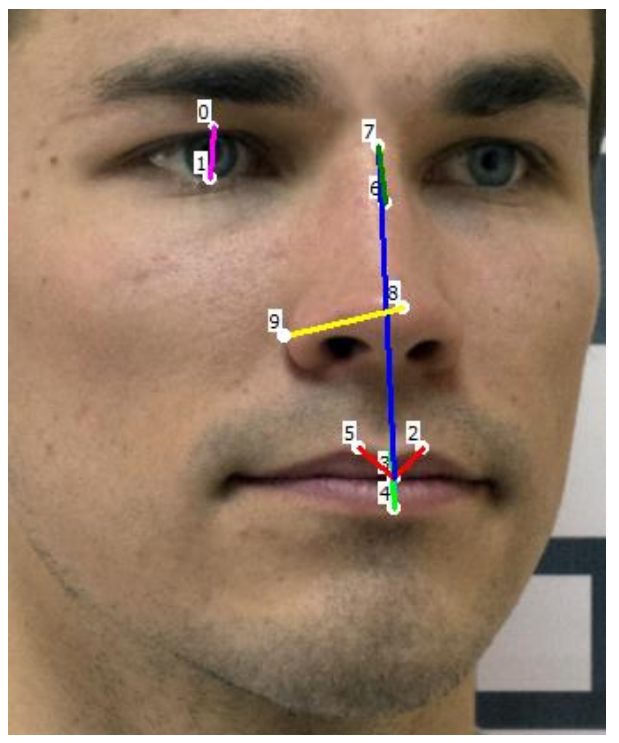

Fig. 2. Part of the image from examination set with placed control points.

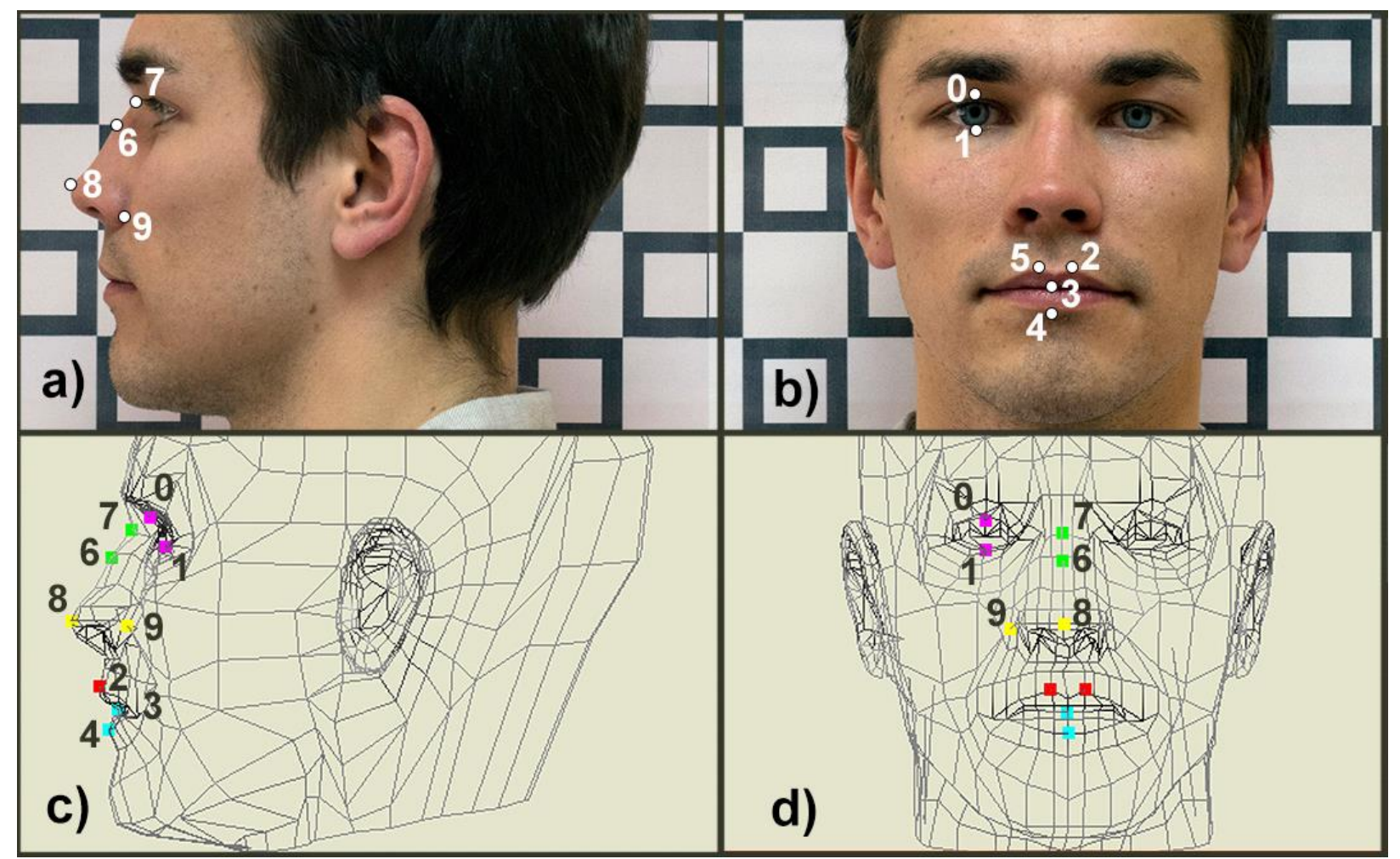

Fig. 3. a), b) Some of the control points on the base images (profile, full face); c), d) Control points that were automatically transferred onto the model of the head. 


\section{3D Models of the Head Positioned on the Image from the Examination Set}

In the next step, each 3D model of the head is automatically positioned on the image of the face (if needed the model is scaled) and the model is rotated to most resemble the rotation angle of the head on the image (or its closest angle).

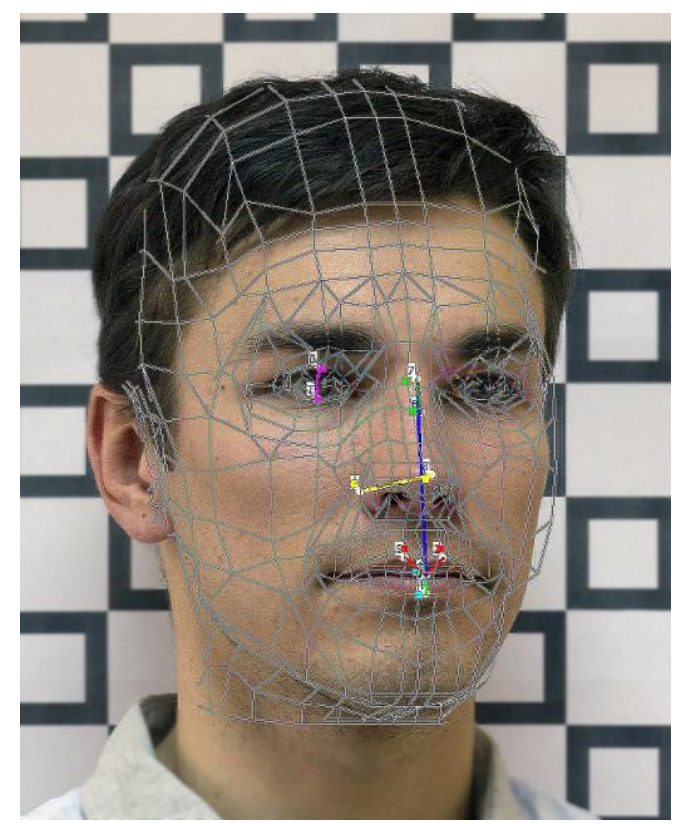

Fig. 4. 3D model positioned on the image from the examination set.

\section{E. Calculating the Distances between the Saved Control Point Coordinates Using Euclidean Distances}

In the next step of the algorithm, in order to compare distances between the control points of the model and control points on the new image from examination set, it is necessary to calculate the Euclidean distances. At first, distances between the control points on image of the model were calculated (see Expression 1). The distances $d_{e}$ on the new image of the face were calculated in a similar way.

$d_{m_{k}}(j)=\frac{1}{h} \sqrt{\left(x_{p+1}(j)-x_{p}(j)\right)^{2}+\left(y_{p+1}(j)-y_{p}(j)\right)^{2}}$

where $j \in[1: m]$ - model number, $m$ - number of models;

$k \in[1: N]$ - distance number, $N$ - number of distances;

$p \in[1: Q]-$ control point index,

$Q$ - number of control points;

$d_{m}(k)$ - distance $(k)$ between the control points of the model (j), pixels;

$x_{p}(j), x_{p+1}(j), y_{p}(j), y_{p+1}(j)$ - coordinates of the control point $(p)$ of the model $(j)$, pixels;

$h$ - height of the image of the model, pixels.

\section{F. Checking the Angle of Rotation of the 3D Model}

In the next step, depending on the angle of rotation of the model, distance numbers were chosen in order to calculate the sum of squares of distances.

Thus, if the angle of rotation of the model was $\alpha>=75$ or $\alpha<=-75$, all distances were used in the calculations otherwise only specific selected distance numbers were used as seen in Table I.

The sums of squares of distances were calculated as follows:

$$
d_{e}(j)=\sum_{k=1}^{N}\left(d_{m k}(j)-d_{e_{k}}\right)^{2}
$$

TABLE I

The Dependency BetweEn ANGLE of Rotation OF THE MODEL AND THE DISTANCE NUMBERS

\begin{tabular}{|l|l|l|}
\hline Interval & $\begin{array}{l}\text { Distance numbers used } \\
\text { in calculations }\end{array}$ & $\begin{array}{l}\text { Distance numbers not } \\
\text { used in calculations }\end{array}$ \\
\hline$(75,90],[-90,-75)$ & $1,2,3,4,5,6,7,8$ & \\
\hline$(60,75],[-75,60)$ & $1,4,7,8$ & $2,3,5,6$ \\
\hline$(45,60]$ & $1,5,7,8$ & $2,3,4,6$ \\
\hline$(15,45]$ & $1,2,3,5$ & $4,6,7,8$ \\
\hline$[-15,15]$ & $1,2,3$ & $4,5,6,7,8$ \\
\hline$[-45,-15)$ & $1,2,3,6$ & $4,5,7,8$ \\
\hline$[-60,-45)$ & $1,6,7,8$ & $2,3,4,5$ \\
\hline
\end{tabular}

TABLE II

DistANCE NuMBERING DEPENDING ON THE CONTROL POINT NUMBERS

\begin{tabular}{|l|l|l|l|l|l|l|l|l|}
\hline Distance No. & 1 & 2 & 3 & 4 & 5 & 6 & 7 & 8 \\
\hline Point No. & 1,0 & 4,3 & 5,4 & 7,3 & 5,3 & 3,2 & 7,6 & 9,8 \\
\hline
\end{tabular}

In the final step, the smallest sum of squares of distances was calculated, which would indicate the class of the image under analysis (see Fig. 5).

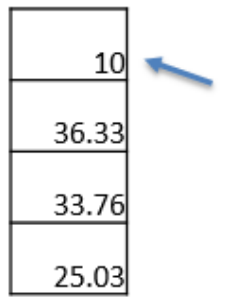

\section{This is a minimal result, therefore the face belongs to the 1 class}

Fig. 5. Example of how the algorithm chooses the class of the face.

\section{EXPERIMENTS}

The images from seven classes ( 7 people) were used in the experiments, with 3 images (see Fig. 5 a) profile, b) half turn, c) profile) in each class that were used to create $3 \mathrm{D}$ models (aee Fig. 5 d) profile, e) half turn, f) profile). The algorithm was tested on 91 images from the examination set (see Fig. 6). To test the algorithm for each class, the rotation angle of the head varied in the range from -90 to 90 degrees with an interval of 15 degrees.

Control points were places on each new image of the face from the examination set and the distances were calculated, the sum of squares and minimum values were also calculated. The algorithm was tested using the extended training set (the model was used), and the base training set (without the model). As can be seen from the results, when the extended training set was used there were no mistakes. In Table III, all the calculated minimum values belonged to the correct classes; however, when only the base training set was used there were 6 mistakes. 
$2015 / 16$

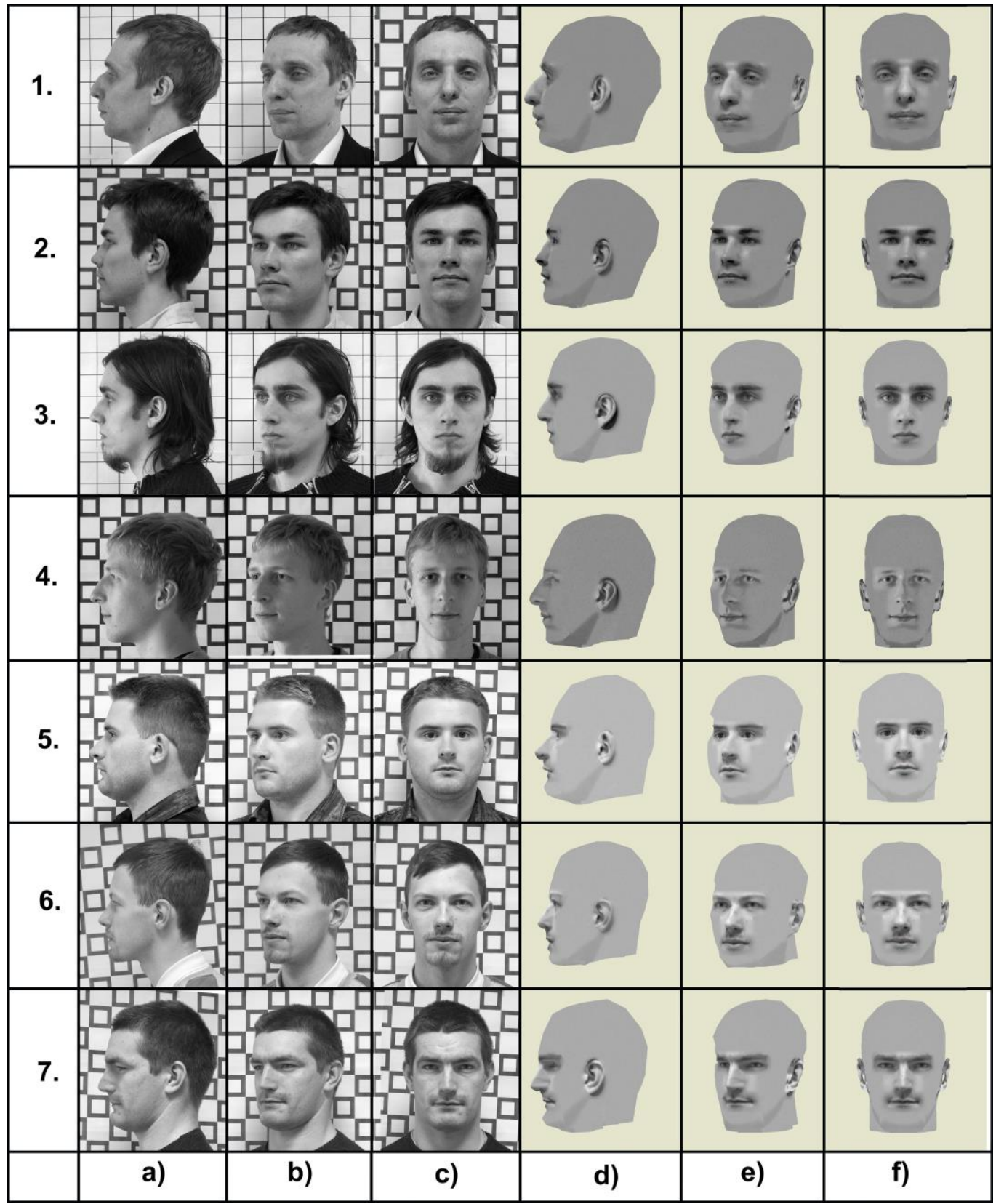

Fig. 6. a), b), c) Training set (base images) of seven classes; d), e), f) Images that match the base images from the training set that were created based on the 3D models. 


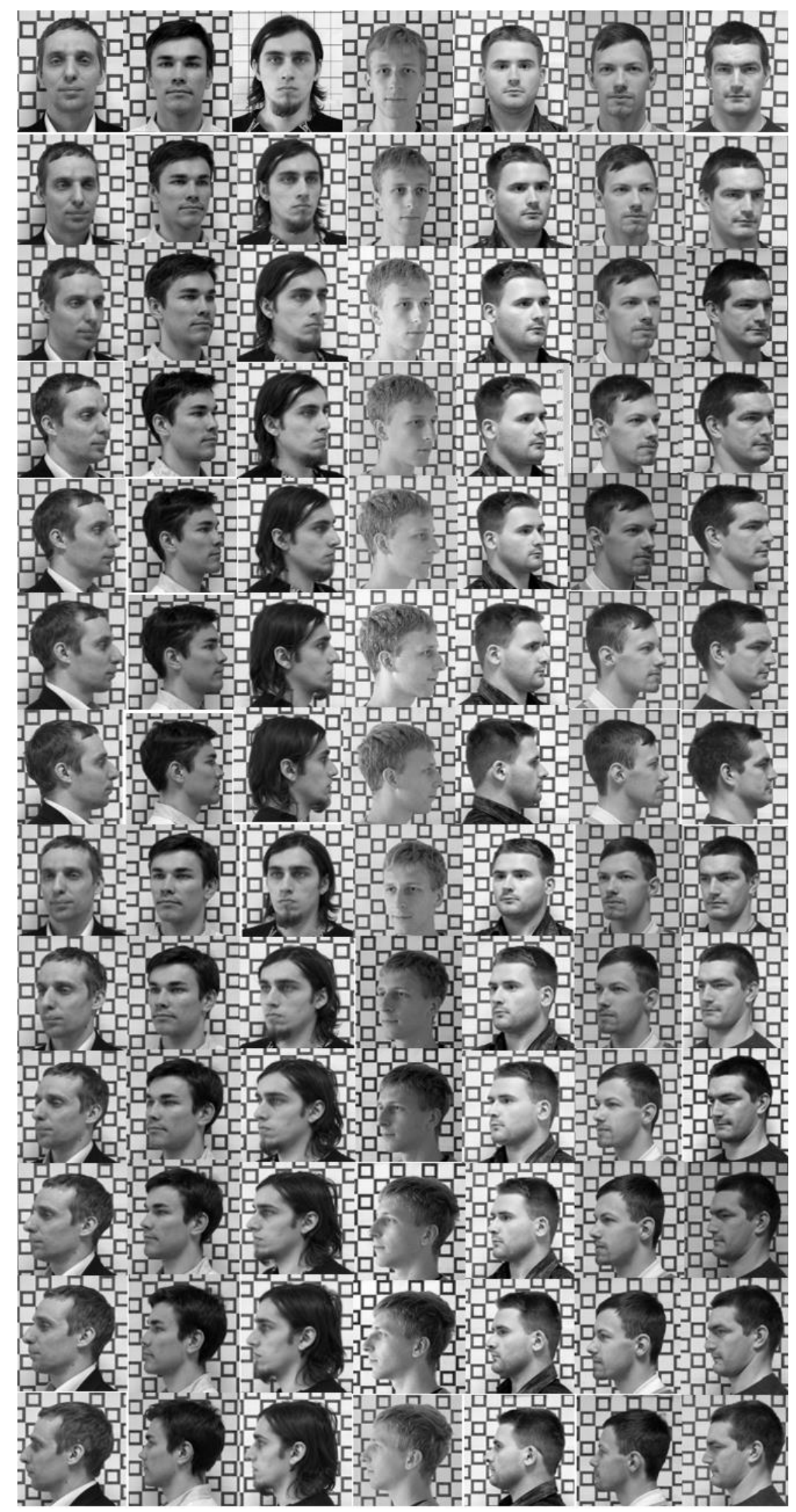

Fig. 7. Examination set that includes 7 classes with 13 images in each class (in total - 91 images). 
TABLE III

RECOGNITION RESUlts FOR SELECTEd DistanCE NUMBERS

\begin{tabular}{|c|c|c|c|c|c|c|c|c|c|c|c|c|c|}
\hline & -90 & -75 & -60 & -45 & -30 & -15 & 0 & 15 & 30 & 45 & 60 & 75 & 90 \\
\hline \multirow[t]{7}{*}{1} & 9.6 & 6.69 & 7.98 & 1.52 & 2.92 & 1.22 & 0.2 & 0.41 & 3.51 & 0.63 & 3.64 & 19.19 & 8.27 \\
\hline & 41.93 & 35.9 & 33.17 & 7.94 & 11.57 & 6.75 & 7.61 & 8.93 & 14.52 & 7.99 & 27.65 & 38.91 & 32.62 \\
\hline & 34.81 & 34.39 & 20.2 & 8.75 & 12.18 & 7.25 & 4.29 & 5.47 & 14.82 & 10.53 & 16.1 & 34.47 & 17.99 \\
\hline & 37.14 & 34.57 & 20.01 & 13.2 & 18.43 & 7.17 & 9.96 & 9.45 & 18.51 & 14.55 & 19.23 & 28.17 & 19.55 \\
\hline & 37 & 41.71 & 35.94 & 9.71 & 16.42 & 4.14 & 3.9 & 4.1 & 17.9 & 11.04 & 30.9 & 40.89 & 30.86 \\
\hline & 39.35 & 33.03 & 26.11 & 12.03 & 18.29 & 9.58 & 7.23 & 10.7 & 17.82 & 12.13 & 21.83 & 33.17 & 26.16 \\
\hline & 23.31 & 32.04 & 20.01 & 13.22 & 15.63 & 9.54 & 11.85 & 12.86 & 19.57 & 18.9 & 16.62 & 21.63 & 16.25 \\
\hline \multirow[t]{7}{*}{2} & 30.37 & 12.18 & 11.11 & 5.83 & 3.76 & 3.21 & 6.12 & 5.71 & 9.87 & 8.99 & 17.56 & 20.51 & 23.57 \\
\hline & 4.32 & 3.77 & 1.08 & 0.81 & 0.39 & 0.38 & 0.45 & 0.29 & 2.1 & 0.65 & 0.37 & 3.61 & 5.78 \\
\hline & 29.3 & 15.62 & 5.39 & 9.22 & 7.5 & 5 & 9.53 & 6.21 & 15.6 & 9.15 & 5.7 & 16.31 & 18.95 \\
\hline & 16.45 & 10.62 & 3.5 & 3.23 & 3.65 & 0.98 & 1.08 & 0.35 & 4.83 & 2.31 & 4.11 & 8.33 & 9.29 \\
\hline & 16.31 & 10.14 & 5.91 & 7.14 & 6.7 & 2.09 & 4.3 & 2.6 & 10.82 & 5.22 & 4.5 & 10.17 & 13.6 \\
\hline & 12.28 & 5.4 & 1.56 & 3.76 & 3.07 & 1.45 & 3.07 & 1.16 & 7.02 & 3.27 & 1.59 & 4.31 & 9.4 \\
\hline & 13.56 & 12.81 & 4.9 & 1.99 & 2.01 & 2.09 & 0.53 & 1.18 & 2.41 & 2.02 & 4.62 & 7.99 & 7.27 \\
\hline \multirow[t]{7}{*}{3} & 11.45 & 12.75 & 4.94 & 1.93 & 2.76 & 1.95 & 2.62 & 3.14 & 1.89 & 3.52 & 4.7 & 13.92 & 12.42 \\
\hline & 22.44 & 17.6 & 12.91 & 5.19 & 6.53 & 5.58 & 5.81 & 9.96 & 5.78 & 5.94 & 7.77 & 13.68 & 15.45 \\
\hline & 9.62 & 8.43 & 3.44 & 0.78 & 0.22 & 0.18 & 0.09 & 0.3 & 1 & 1.5 & 3.66 & 5.58 & 4.76 \\
\hline & 14.85 & 14.43 & 7.07 & 9.17 & 8.63 & 5.34 & 6.26 & 9.9 & 5.85 & 9 & 4.45 & 11.12 & 7.72 \\
\hline & 10.93 & 13.07 & 11.7 & 4.62 & 4.74 & 1.49 & 1.6 & 3.65 & 3.18 & 4.59 & 9.4 & 12.62 & 12.31 \\
\hline & 15.64 & 10.31 & 11.89 & 4.6 & 5.97 & 3.29 & 2.21 & 6.53 & 3.1 & 3.94 & 4.57 & 6.84 & 7.97 \\
\hline & 18 & 18.62 & 5.07 & 11.49 & 10.67 & 9.23 & 10.89 & 15.01 & 9.31 & 13.89 & 7.46 & 17.26 & 12.3 \\
\hline \multirow[t]{7}{*}{4} & 27.6 & 14.01 & 12.66 & 13.99 & 7.37 & 6.14 & 5.54 & 6.19 & 8.71 & 7.76 & 6.49 & 25.04 & 25.04 \\
\hline & 17.59 & 16.7 & 5.63 & 3.15 & 0.9 & 1.53 & 5.73 & 0.41 & 1.5 & 0.7 & 8.22 & 15.08 & 15.08 \\
\hline & 17.01 & 23.11 & 8.52 & 8.24 & 5.71 & 4.57 & 10.67 & 6.77 & 6.72 & 5.14 & 7.31 & 18.18 & 18.18 \\
\hline & 2.48 & 9.45 & 0.95 & 0.31 & 0.42 & 0.35 & 5.1 & 0.19 & 0.15 & 0.68 & 3.82 & 3.04 & 3.04 \\
\hline & 15.1 & 23.69 & 11.06 & 2.98 & 2.33 & 1.48 & 3.78 & 2.64 & 2.1 & 1.95 & 13.77 & 18.42 & 18.42 \\
\hline & 7.31 & 10.17 & 1.53 & 2.42 & 1.22 & 0.8 & 8.05 & 1.44 & 1.22 & 1.22 & 4.46 & 7.04 & 7.04 \\
\hline & 5.05 & 13.46 & 5.03 & 1.65 & 1.38 & 1.66 & 4.51 & 0.86 & 1.13 & 1.65 & 4.47 & 6.18 & 6.18 \\
\hline \multirow[t]{7}{*}{5} & 39.1 & 24.62 & 23.43 & 8.35 & 8.21 & 3.97 & 3.5 & 4.43 & 10.34 & 15.39 & 28.47 & 28.13 & 35.61 \\
\hline & 14.35 & 8.85 & 5.9 & 6.87 & 4.14 & 3.56 & 3.71 & 3.57 & 6.33 & 8.65 & 5.58 & 6.11 & 6.72 \\
\hline & 27.81 & 18.32 & 4.53 & 12.26 & 7.73 & 7.34 & 6.57 & 7.13 & 5.85 & 4.35 & 6.09 & 12.01 & 17.15 \\
\hline & 13.58 & 11.46 & 6.7 & 6.75 & 2.14 & 2.14 & 3.18 & 2.03 & 1.5 & 3.39 & 9.96 & 7.13 & 9.69 \\
\hline & 12.56 & 7.6 & 1.47 & 6.2 & 1.47 & 1.85 & 1.5 & 1.68 & 0.83 & 1.86 & 0.89 & 4.87 & 5.92 \\
\hline & 20.95 & 13.24 & 9.78 & 10.98 & 7.1 & 5.73 & 4.65 & 5.52 & 3.55 & 3.85 & 9.16 & 8.95 & 10.92 \\
\hline & 12.57 & 12.23 & 9.1 & 6.67 & 4.2 & 3.29 & 3.81 & 3.26 & 4.56 & 6.6 & 13.23 & 5.51 & 11.97 \\
\hline \multirow[t]{7}{*}{6} & 23.6 & 14.05 & 21.39 & 5.3 & 5.81 & 6.74 & 7.79 & 3.88 & 4.27 & 5.7 & 13.27 & 13.02 & 24.29 \\
\hline & 7.67 & 7 & 3.02 & 1.34 & 3.78 & 1.39 & 4.94 & 1.94 & 2.28 & 4.04 & 1.14 & 6.2 & 9.12 \\
\hline & 21.85 & 15.63 & 9.35 & 4.05 & 5.07 & 5.45 & 4.26 & 2.54 & 4.9 & 3.8 & 5.85 & 9.16 & 14.41 \\
\hline & 10.12 & 10.37 & 0.86 & 4.86 & 8.28 & 2.93 & 6.75 & 2.76 & 5.91 & 8.38 & 3.02 & 6.54 & 17.53 \\
\hline & 14 & 11.11 & 7.41 & 6.47 & 9.99 & 4.98 & 6.93 & 2.51 & 7.02 & 6.39 & 6.35 & 6.96 & 8.72 \\
\hline & 6.93 & 6.95 & 0.7 & 0.99 & 2.12 & 0.5 & 2.16 & 0.83 & 2.22 & 3.26 & 0.22 & 3.48 & 8.68 \\
\hline & 11.77 & 12.34 & 4.74 & 5.52 & 8.22 & 4.26 & 10.98 & 5.17 & 6.57 & 11.35 & 6.05 & 8.18 & 25.27 \\
\hline \multirow[t]{7}{*}{7} & 75.07 & 21.47 & 15.88 & 25.49 & 22.75 & 12.69 & 17.65 & 16.01 & 26.39 & 17.7 & 20.53 & 28.62 & 38.77 \\
\hline & 32.12 & 10.3 & 5.01 & 8.61 & 8.26 & 2.76 & 5.06 & 3.25 & 7.02 & 5.74 & 5.22 & 8.86 & 8.5 \\
\hline & 51.72 & 19.19 & 4.54 & 26.26 & 28.89 & 17.34 & 22.52 & 19.77 & 29.34 & 22.46 & 6.07 & 17.4 & 21.73 \\
\hline & 17.05 & 5.43 & 1.23 & 5.97 & 8.36 & 2.94 & 4.33 & 3.41 & 7.91 & 7.86 & 3.96 & 6.78 & 5.53 \\
\hline & 36.53 & 13.93 & 5.94 & 16.92 & 18.21 & 9.05 & 12.65 & 11.06 & 18 & 14.51 & 6.19 & 8.2 & 14.34 \\
\hline & 30.72 & 8.41 & 3.71 & 11.98 & 13.16 & 7.01 & 10.5 & 7.58 & 13.38 & 12.38 & 6.6 & 10.72 & 9.72 \\
\hline & 16.16 & 4.52 & 0.59 & 3.25 & 4.46 & 1.13 & 1.36 & 1.32 & 3.47 & 4.95 & 0.73 & 3.36 & 5.47 \\
\hline
\end{tabular}


TABLE IV

RECOGNITION RESULTS FOR ALL DISTANCE NUMBERS

\begin{tabular}{|c|c|c|c|c|c|c|c|c|c|c|c|c|c|}
\hline & -90 & -75 & -60 & -45 & -30 & -15 & 0 & 15 & 30 & 45 & 60 & 75 & 90 \\
\hline \multirow[t]{7}{*}{1} & 9.6 & 6.69 & 8.22 & 8.1 & 12.25 & 11.2 & 7.8 & 11.33 & 6.84 & 6.83 & 4.82 & 19.19 & 8.27 \\
\hline & 41.93 & 35.9 & 35.88 & 31.48 & 40.46 & 45.05 & 41.47 & 51.54 & 32.49 & 37.64 & 30.14 & 38.91 & 32.62 \\
\hline & 34.81 & 34.39 & 28.59 & 26.81 & 40.12 & 44.09 & 37.27 & 47.1 & 34.69 & 34.14 & 24.46 & 34.47 & 17.99 \\
\hline & 37.14 & 34.57 & 30.46 & 28.86 & 41.13 & 49.68 & 49.47 & 54.96 & 34.27 & 37.79 & 28.06 & 28.17 & 19.55 \\
\hline & 37 & 41.71 & 42.72 & 41.74 & 57.19 & 63.21 & 57.97 & 66.57 & 47.96 & 49.14 & 36.15 & 40.89 & 30.86 \\
\hline & 39.35 & 33.03 & 32.47 & 28.05 & 39.23 & 42.91 & 36.29 & 47.38 & 29.63 & 33.16 & 28.54 & 33.17 & 26.16 \\
\hline & 23.31 & 32.04 & 30.22 & 30.14 & 42.42 & 46.56 & 39.79 & 53.64 & 39.27 & 40.15 & 27.05 & 21.63 & 16.25 \\
\hline \multirow[t]{7}{*}{2} & 30.37 & 12.18 & 13.31 & 17.7 & 12.02 & 10.78 & 14.77 & 8.89 & 19.35 & 22.13 & 24.4 & 20.51 & 23.57 \\
\hline & 4.32 & 3.77 & 1.13 & 3.5 & 4.65 & 6.73 & 2.64 & 4.88 & 3.12 & 3.2 & 1.84 & 3.61 & 5.78 \\
\hline & 29.3 & 15.62 & 9.28 & 13.81 & 13.63 & 14.19 & 15.96 & 9.72 & 18.3 & 12.94 & 11.36 & 16.31 & 18.95 \\
\hline & 16.45 & 10.62 & 6.69 & 4.18 & 6.54 & 12.48 & 7.7 & 5.92 & 5.62 & 3.21 & 5.56 & 8.33 & 9.29 \\
\hline & 16.31 & 10.14 & 8.37 & 12.9 & 14.72 & 18.35 & 15.6 & 15.05 & 14.57 & 10.88 & 7.85 & 10.17 & 13.6 \\
\hline & 12.28 & 5.4 & 3.08 & 5.15 & 5.84 & 7.43 & 5.4 & 4.74 & 7.24 & 3.92 & 4.78 & 4.31 & 9.4 \\
\hline & 13.56 & 12.81 & 7.97 & 8.22 & 8.01 & 11.04 & 9.4 & 5.96 & 6.34 & 8.59 & 6.91 & 7.99 & 7.27 \\
\hline \multirow[t]{7}{*}{3} & 11.45 & 12.75 & 7.75 & 10.58 & 9.99 & 6.62 & 6.08 & 6.62 & 7.11 & 10.07 & 12.75 & 13.92 & 12.42 \\
\hline & 22.44 & 17.6 & 15.01 & 13.58 & 12.48 & 8.07 & 9.67 & 19.65 & 13.42 & 10.44 & 12.13 & 13.68 & 15.45 \\
\hline & 9.62 & 8.43 & 4.6 & 5.79 & 5.96 & 3.33 & 3.55 & 9.87 & 7.1 & 3.36 & 3.95 & 5.58 & 4.76 \\
\hline & 14.85 & 14.43 & 12.57 & 13.9 & 12.77 & 11.74 & 13.49 & 24.05 & 10.78 & 10.91 & 8.47 & 11.12 & 7.72 \\
\hline & 10.93 & 13.07 & 13.35 & 15.62 & 14.85 & 11.33 & 13.19 & 25.56 & 16.89 & 11.56 & 11.78 & 12.62 & 12.31 \\
\hline & 15.64 & 10.31 & 13.16 & 10.61 & 9.87 & 5.63 & 4.73 & 14.67 & 7.24 & 5.56 & 5.47 & 6.84 & 7.97 \\
\hline & 18 & 18.62 & 12.33 & 15.78 & 16.04 & 12.56 & 14.37 & 25.91 & 15.44 & 16.11 & 14.32 & 17.26 & 12.3 \\
\hline \multirow[t]{7}{*}{4} & 27.6 & 14.01 & 16.21 & 16.47 & 11.34 & 17.04 & 25.76 & 13.7 & 14.04 & 16.61 & 9.04 & 25.04 & 25.04 \\
\hline & 17.59 & 16.7 & 5.77 & 12.23 & 12.27 & 6.29 & 9.37 & 2.95 & 13.03 & 8.84 & 8.56 & 15.08 & 15.08 \\
\hline & 17.01 & 23.11 & 12.54 & 13.18 & 16.03 & 9.79 & 13.37 & 9.11 & 18.33 & 10.08 & 9.74 & 18.18 & 18.18 \\
\hline & 2.48 & 9.45 & 2.65 & 6.83 & 7 & 1 & 8.31 & 3.21 & 6.61 & 2.45 & 6.34 & 3.04 & 3.04 \\
\hline & 15.1 & 23.69 & 12.59 & 17.13 & 21.5 & 9.85 & 4.63 & 11.24 & 22 & 13.54 & 14.69 & 18.42 & 18.42 \\
\hline & 7.31 & 10.17 & 2.9 & 7.94 & 7.12 & 2.07 & 12.39 & 3.03 & 6.77 & 4.28 & 5.6 & 7.04 & 7.04 \\
\hline & 5.05 & 13.46 & 6.85 & 6.27 & 12.15 & 9.86 & 9.33 & 5.55 & 14.15 & 7.39 & 8.51 & 6.18 & 6.18 \\
\hline \multirow[t]{7}{*}{5} & 39.1 & 24.62 & 36.08 & 28.87 & 22.49 & 28.45 & 22.89 & 17.68 & 34.28 & 45.64 & 44.53 & 28.13 & 35.61 \\
\hline & 14.35 & 8.85 & 11.8 & 9.31 & 5.16 & 6.94 & 5.96 & 4.57 & 12.95 & 13.73 & 11.83 & 6.11 & 6.72 \\
\hline & 27.81 & 18.32 & 21.45 & 16.44 & 11.16 & 13.2 & 9.06 & 8.29 & 9.49 & 13.17 & 18.99 & 12.01 & 17.15 \\
\hline & 13.58 & 11.46 & 11.32 & 9.99 & 7.33 & 8.45 & 6.86 & 4.37 & 5.95 & 12.8 & 12.69 & 7.13 & 9.69 \\
\hline & 12.56 & 7.6 & 9.96 & 8.17 & 2.39 & 4.4 & 2.5 & 4.94 & 4.98 & 4.91 & 9.96 & 4.87 & 5.92 \\
\hline & 20.95 & 13.24 & 20.21 & 14.86 & 11.25 & 11.56 & 8.38 & 7.07 & 10.81 & 12.41 & 17.79 & 8.95 & 10.92 \\
\hline & 12.57 & 12.23 & 12.12 & 12.45 & 7.5 & 9.75 & 8.8 & 6.21 & 9.75 & 17.42 & 15.44 & 5.51 & 11.97 \\
\hline \multirow[t]{7}{*}{6} & 23.6 & 14.05 & 22.18 & 15.35 & 9.03 & 16.98 & 9.38 & 5.41 & 10.26 & 9.84 & 17.69 & 13.02 & 24.29 \\
\hline & 7.67 & 7 & 4.06 & 5.61 & 9.52 & 6.53 & 10.17 & 7.98 & 4.13 & 7.13 & 5.21 & 6.2 & 9.12 \\
\hline & 21.85 & 15.63 & 12.89 & 9.02 & 12.08 & 14.71 & 10.53 & 8.28 & 9.47 & 5.31 & 6.71 & 9.16 & 14.41 \\
\hline & 10.12 & 10.37 & 6.88 & 7.83 & 13.97 & 12.36 & 17.49 & 10.9 & 7.89 & 12.44 & 10.01 & 6.54 & 17.53 \\
\hline & 14 & 11.11 & 10.16 & 13.21 & 21.29 & 18.69 & 23.11 & 18.33 & 13.84 & 12.45 & 8.64 & 6.96 & 8.72 \\
\hline & 6.93 & 6.95 & 3.07 & 4.64 & 6.17 & 5.49 & 6.77 & 5.76 & 2.51 & 4.97 & 2.01 & 3.48 & 8.68 \\
\hline & 11.77 & 12.34 & 11.44 & 11.19 & 14.22 & 13.84 & 16.25 & 11.8 & 11.89 & 13.26 & 16.32 & 8.18 & 25.27 \\
\hline \multirow[t]{7}{*}{7} & 75.07 & 21.47 & 28.98 & 40.56 & 33.19 & 20.08 & 35.63 & 24.65 & 39.02 & 25.42 & 32.03 & 28.62 & 38.77 \\
\hline & 32.12 & 10.3 & 11.04 & 9.66 & 9.16 & 3.63 & 7.86 & 4.06 & 7.83 & 8.43 & 8.79 & 8.86 & 8.5 \\
\hline & 51.72 & 19.19 & 21.65 & 30.05 & 30.66 & 20.41 & 27.02 & 21.22 & 32.37 & 25.47 & 14.81 & 17.4 & 21.73 \\
\hline & 17.05 & 5.43 & 5.82 & 7.76 & 9.29 & 6.32 & 7.86 & 5.72 & 9.05 & 9.1 & 5.37 & 6.78 & 5.53 \\
\hline & 36.53 & 13.93 & 14.86 & 19.5 & 20.65 & 16.15 & 15.74 & 16.61 & 20.68 & 20.93 & 12.02 & 8.2 & 14.34 \\
\hline & 30.72 & 8.41 & 14.21 & 13.61 & 14.17 & 7.81 & 13.96 & 8.44 & 14.25 & 12.57 & 11.99 & 10.72 & 9.72 \\
\hline & 16.16 & 4.52 & 3.42 & 9.1 & 6.76 & 5.26 & 8.22 & 4.5 & 7.61 & 9.48 & 2.26 & 3.36 & 5.47 \\
\hline
\end{tabular}

\section{CONCLUSION}

In Table III, every image from the examination set has a corresponding rotation angle (from -90 to 90 degrees). The table is divided into 4 blocks, each containing 4 rows, where the blocks describe different classes (people) and each row describes a different model. The first block contains the distances of the examination face from the 1 class for all 7 models and the row with the minimum distances is highlighted. In this table, the selected distances were calculated depending on the angle of rotation of the head. As can be seen from Table III, the minimum distances are consistent with the images from the examination set.
In Table IV, all distances were calculated for equal angles of rotation. As can be seen from Table IV, using all distances does not produce good enough recognition results, because there are 9 errors in different angles of rotation of the head.

As results show, it is necessary to calculate specific distance numbers at different angles of rotation of the head, which allows decreasing the number of errors and improving the results of the new face recognition algorithm. This algorithm could be used in forensics, when only a limited training set is available. An expert could build a model based on the information that was acquired from base images and later use it in the recognition process, which could significantly decrease the time of recognition. 


\section{REFERENCES}

[1] T. Kim, J. Kittler, "Locally linear discriminant analysis for multimodally distributed classes for face recognition", IEEE Transactions on Pattern Analysis and Machine Intelligence, vol. 27, Issue 3, 2005, pp. 318-327. http://dx.doi.org/10.1109/TPAMI.2005.58

[2] R. Brunelli and T. Poggio, "Face recognition: features versus templates", IEEE Transactions on Pattern Analysis and Machine Intelligence, vol. 15, no 10, 1993, pp. 1042-1052. http://dx.doi.org/10.1109/34.254061

[3] Y. Li, S. Gong, H. Liddell, "Support vector regression and classification based multiview face detection and recognition", Int. Conf. on Face and Gesture Recognition, 2000, pp. 300-305.

[4] T. Vetter, V. Blanz, "A morphable model for the synthesis of 3D faces", Proc. SIGGRAPH '99 Proc. of the 26th annual conf. on Computer graphics and interactive techniques', 1999, pp. 187-194. http://dx.doi.org/10.1145/311535.311556

[5] V. Blanz, S. Romdhani, T. Vetter, "Face identification across different poses and illumination with a 3D morphable model", Int. Conf. on Automatic Face and Gesture Recognition, 2002, pp. 202-207.

[6] I. Kemelmacher-Shlizerman and R. Basri, "3d face reconstruction from a single image using a single reference face shape," IEEE Transactions on Pattern Analysis and Machine Intelligence, vol. 33, no. 2, 2011, pp. 394-405. http://dx.doi.org/10.1109/TPAMI.2010.63

[7] B. Horn and M. Brooks, "The variational approach to shape from shading," Computer Vision, Graphics, and Image Processing, vol. 33, no. 2, 1986, pp. 174-208. http://dx.doi.org/10.1016/0734-189X(86)90114-3

[8] J. Huang, B. Heisele, and V. Blanz, "Component-based face recognition with 3d morphable models," in Audio-and Video-Based Biometric Person Authentication. Springer, 2003, pp. 1055-1055.

[9] V. Blanz and T. Vetter, "Face recognition based on fitting a 3d morphable model," IEEE Transactions on Pattern Analysis and Machine

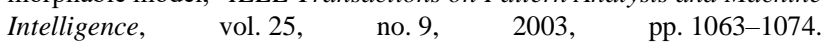
http://dx.doi.org/10.1109/TPAMI.2003.1227983

[10] Y. Taigman and L. Wolf, "Leveraging billions of faces to overcome performance barriers in unconstrained face recognition," Arxiv preprint arXiv, 2011, pp. 1108-1122.

[11] L. Wolf, T. Hassner, and Y. Taigman, "Effective unconstrained face recognition by combining multiple descriptors and learned background statistics," IEEE Transactions on Pattern Analysis and Machine Intelligence, vol. 33, no. 10, $2011 \quad$ pp. 1978-1990. http://dx.doi.org/10.1109/TPAMI.2010.230

[12] G. Huang, M. Mattar, T. Berg, E. Learned-Miller et al., "Labeled faces in the wild: A database for studying face recognition in unconstrained environments," 2008.

[13] O. Krutikova, A. Glazs, "Development of a New Method for Adapting a 3D Model from a Minimum Number of 2D Images", Technologies of Computer Control, 2013, vol. 14, pp. 12-17.

[14] O. Krutikova, A. Glazs, "Semi-automatic method of face recognition based on extended training set", in Proc. of Int. Conf. Biomedical Engineering, Lithuania, Kaunas, 2014, pp. 148-152.

[15] O. Krutikova, A. Glazs, "Increasing the training set in face recognition tasks by using a 3D model of a face", RTU Zinātniskie raksti Datorvadības tehnologijas, vol. 15, 2014, pp. 14-19. http://dx.doi.org/10.7250/tcc.2014.002

Olga Krutikova was born in Riga, Latvia, on 8 January 1988. She is a PhD student at Riga Technical University, Faculty of Computer Science and Information Technology.

She received the degree of Master of Engineering Sciences from Riga

Technical University in 2012.

E-mail: Olga.Krutikova@ rtu.lv

Aleksandrs Glazs was born in Riga, Latvia, on 7 April 1939. He is a Professor at Riga Technical University, Faculty of Computer Science and Information Technology, Vice-Director of the Institute of Computer Control, Automation and Computer Engineering, Head of the Department of Image Processing and Computer Graphics.

He received the degree of Candidate of Technical Sciences from Riga Polytechnic Institute in 1971 and the degree of Doctor of Technical Sciences (Dr. habil. sc. ing.) from the Russian Academy of Sciences in 1992. He has more than 100 scientific publications in different areas: pattern recognition, image processing, computer vision and computer graphics.

A. Glazs is a full member of the Baltic Informatization Academy.

E-mail: glaz@egle.cs.rtu.lv

Olga Krutikova, Aleksandrs Glazs. Pusautomātiskā sejas atrašanas metode, kura balstās uz 3D modẹ̣a, nezināmās sejas atpazīšanai

Seju atpazišana ir viens no aktuālākajiem virzieniem tēlu atpazǐšanas teorijā. To plaši lieto dažādās dzīves sfērās, piemēram, kriminālistikā bieži ir nepieciešams identificēt personu, balstoties tikai uz bāzes attēliem (pretskats, pagrieziens, sānskats). Viena no galvenajām problēmām tēlu atpazīšanā ir nepietiekamais bāzes apmācošās izlases apjoms. Šo problēmu var atrisināt, lietojot 3D modeli, pagriežot to dažādos lenkosos, tādejādi iegūstot papildus informāciju un papildinot sākotnējo apmācošu izlasi.

Dotajā rakstā tiek piedāvāts pusautomātiskais seju atpazišanas algoritms gadījumam, kad ir nepietiekama apmācošā izlase (pretskats, pagrieziens, sānskats). Atpazī̌anas algoritmā tiek lietots poligonālais galvas 3D modelis, kas tika izveidots, balstoties uz informāciju no bāzes attēliem. Sejas tiek salīdzinātas, analizējot attālumus, kas tika iegūti ar kontrolpunktu palīdzību. No sākuma kontrolpunkti tiek pārnesti no bāzes attēliem uz 3D modeḷiem, ka arī tiek izvietoti uz jaunā attēla no eksaminācijas izlases. Tālāk modelis tiek lietots, lai noteiktu galvas pagrieziena lenkịi jaunajā attēlā. Tiek izskaitḷti attālumi starp kontrolpunktiem gan modeḷu attēlos, gan jaunajā attēlā, analizējot datus, tiek noteikta klase, kurai pieder seja.

Šo algoritmu var lietot kriminālistikā, kur sākotnēja informācija par sejas formu ir strikti ierobežota un ir pieejami tikai 3 cilvēka attēli. Ar šĩ algoritma palīdzību var izveidot galvas 3D modeli un atpazīt seju jaunajā attēlā, kas paātrinātu eksperta darbu un samazinātu nepareizas atpazišanas kḷūdu skaitu.

Ольга Крутикова, Александр Глаз. Полуавтоматический метод нахождения изображения лица, который основывается на 3D модели, для распознавания неизвестного лица

Распознавание лиц является одним из актуальных направлений в теории распознавания образов. Его широко применяют в различных

сферах, например, в криминалистике, когда необходимо идентифицировать персону на основе данных, полученных с базовых изображений (анфас, поворот, профиль). Одна из главных проблем распознавания образов, - недостаточный объем начальной обучающей выборки. Эту проблему можно решить при помощи 3D модели, поворачивая ее под различными углами, получая таким образом дополнительную информацию и расширяя начальную обучающую выборку.

В данной статье предлагается полуавтоматический алгоритм распознавания лиц для случая, когда не имеется достаточной обучающей выборки (анфас, поворот, профиль). В алгоритме распознавания используется полигональная 3D модель головы, которая получена, на основе информации, полученной с базовых изображений. Лица сравниваются после анализа расстояний, полученных при помощи контрольных точек. С начала контрольные точки переносятся с базовых изображений на 3D модели, а также размещаются на новом изображении лица из экзаменационного класса. Далее модель применяется для определения поворота головы на новом изображении лица. Рассчитываются расстояния между контрольными точками на изображениях моделей, а также на новом изображении лица и, анализируя данные, определяется класс, к которому принадлежит лицо.

Данный алгоритм можно применять в криминалистике, где начальная информация о форме лица существенно ограничена, и доступны только 3 изображения лица. С помощью алгоритма можно построить $3 \mathrm{D}$ модель и распознать новое лицо, что значительно ускорило бы работу эксперта и уменьшило бы количество ошибок в процессе распознавания. 